


\title{
Comparison of Dosimetrical and Radiobiological Parameters on Three VMAT Techniques for Left-Sided Breast Cancer
}

\author{
Seong-Hee Kang ${ }^{1}$, Jin-Beom Chung ${ }^{1}$, Kyung-Hyeon Kim², Sang-Won Kang ${ }^{2}$, Keun-Yong Eom ${ }^{1}$, \\ Changhoon Song ${ }^{1}$, In-Ah Kim ${ }^{1}$, Jae-Sung Kim ${ }^{1}$ \\ ${ }^{1}$ Department of Radiation Oncology, Seoul National University Bungdang Hospital, Seongnam, ${ }^{2}$ Department of Biomedicine and Health \\ Sciences, Research Institute of Biomedical Engineering, College of Medicine, The Catholic University of Korea, Seoul, Korea
}

Received 12 December 2018 Revised 27 December 2018

Accepted 17 January 2019

Corresponding author

Jin-Beom Chung

(jbchung1213@gmail.com)

Tel: 82-31-787-7654

Fax: 82-31-787-4019
Purpose: To compare the dosimetrical and radiobiological parameters among various volumetric modulated arc therapy (VMAT) techniques using restricted and continuous arc beams for left-sided breast cancer.

Materials and Methods: Ten patients with left-sided breast cancer without regional nodes were retrospectively selected and prescribed the dose of 42.6 Gy in 16 fractions on the planning target volume (PTV). For each patient, three plans were generated using the Eclipse ${ }^{\mathrm{TM}}$ system (Varian Medical System, Palo Alto, CA) with one partial arc 1pVMAT, two partial arcs 2pVMAT, and two tangential arcs 2tVMAT. All plans were calculated through anisotropic analytic algorithm and photon optimizer with $6 \mathrm{MV}$ photon beam of VitalBEAM ${ }^{\mathrm{TM}}$. The same dose objectives for each plan were used to achieve a fair comparison during optimization.

Results: For PTV, dosimetrical parameters such as Homogeneity index, conformity index, and conformal number were superior in $2 \mathrm{pVMAT}$ than those in both techniques. $\mathrm{V}_{95 \%}$, which indicates PTV coverage, was $91.86 \%, 96.60 \%$, and $96.65 \%$ for $1 \mathrm{pVMAT}$, 2pVMAT, and 2tVMAT, respectively. In most organs at risk (OARs), 2pVMAT significantly reduced the delivered doses compared with the other techniques, excluding the doses to contralateral lung. For the analysis of radiobiological parameters, a significant difference in normal tissue complication probability was observed in ipsilateral lung while no difference was observed in the other OARs.

Conclusions: Our study showed that 2pVMAT had better plan quality and normal tissue sparing than $1 \mathrm{pVMAT}$ and 2tVMAT but not for all parameters. Therefore, 2pVMAT could be considered the priority choice for the treatment planning for left breast cancer.

Keywords: Left sided breast cancer, Volumetric modulated arc therapy, Dosimetrical parameters, Radiobiological parameters

\section{Introduction}

Adjuvant radiation therapy (RT) after breast conserving surgery which is the standard of care for early stage breast cancer has been mainly performed with $3 \mathrm{D}$ conformal radiation therapy (3D CRT) using tangential fields. ${ }^{1-3)}$ It is possible for the 3D CRT to provide adequate target coverage with relatively low complication rates. ${ }^{3)}$ However, normal tissue complications such as radiation pneumonitis and heart disease remain a concern. ${ }^{4-6)}$ There is mounting 
evidence that even small delivered doses to the heart during RT are important in the long term survival. ${ }^{6)}$ In particular, left-sided breast cancer with concave shape is difficult to deliver the prescribed dose adequately without irradiation to portion of the lung and heart with 3D CRT. ${ }^{7)}$ During left sided breast RT, it is important to reduce the delivered dose to heart because patients could receive a relatively high cardiac dose which is associated with an increasing risk for heart complications. ${ }^{8}$ In the study reported Darby et al. ${ }^{9}$, the delivered heart dose to breast RT was increased the rate of major coronary events by $7.4 \%$ per Gy.

Dose inhomogeneity which is the predictor of radiationinduced toxicity can be increased by hot-spots within both target and surrounding normal tissues of large breast. Intensity-modulated radiation therapy (IMRT) which allows a homogeneous dose distribution to target has been used to mitigate normal tissue complication. ${ }^{10,11)} \mathrm{Xu}$ et al. ${ }^{12)}$ reported that IMRT could significantly reduce heart dose in case of clinical target volume (CTV) more than $500 \mathrm{cc}$ compared with conventional 3D CRT. However, several studies showed that IMRT using multiple fields increases the irradiated low-dose volumes in contralateral breast and both lungs. ${ }^{13,14)}$ In recent studies, volumetric modulated arc therapy (VMAT) technique has also been compared with various techniques such as 3D CRT and IMRT. ${ }^{14,15)}$ Badakhshi et al. ${ }^{16)}$ reported that the VMAT using two arcs was inferior to IMRT and 3D CRT for the dose distributions in organs at risk (OARs), especially for low doses and mean dose. However, doses to heart and ipsilateral lung could be reduced by VMAT using restricted tangential angles although the dose distribution on target was not improved. ${ }^{17,18)}$

In this work, we compared various VMAT techniques using restricted arc beam and continuous arc beams to the left sided breast cancer to evaluate the delivered doses to OARs and dose homogeneity within the target volume. In addition, radiobiological parameters in lung and heart were analyzed to these techniques.

\section{Materials and Methods}

\section{Patient selection and contouring}

A total of ten patients with left-sided breast cancer without regional nodes who underwent breast-conserving surgery for T0/T1 invasive ductal carcinoma were selected in this retrospective study. The mean age of the patients was 51 years (range, 41 to 70 years). CT simulation was performed with Brilliance CT Big Bore ${ }^{\mathrm{TM}}$ (Philips, USA) with 5-mm slice thickness. All patients were immobilized with the breast board (CIVICO Medical Solutions, USA) in a supine position. The images were transferred to treatment planning system (Eclipse, v. 13.7, Varian Medical System, USA). For each patient, the tumor bed was delineated as clinical target volume (CTV) which includes glandular breast tissue cropped $5 \mathrm{~mm}$ inside the body contour, and the planning target volume (PTV) was defined as the CTV plus a treatment margin of 10 $\mathrm{mm}$ for superior-inferior, $7 \mathrm{~mm}$ for anterior-posterior, and 5 $\mathrm{mm}$ for left-right to allow set-up uncertainties and account for respiratory motion. The breast PTVs ranged from 378 to $1,400 \mathrm{cc}(775 \pm 300 \mathrm{cc})$. The OARs defined in heart, ipsilateral lung, and contralateral lung.

\section{Dose prescription and objective}

The dose of 42.6 Gy in 16 fractions was prescribed to the PTV as the Ontario Canadian trial. ${ }^{19)}$ The plan objectives are summarized in Table 1. The primary goal for planning was to cover at least $100 \%$ of the PTV with $95 \%$ of the prescribed dose to ensure dose coverage of target volume. For PTV homogeneity, $107 \%$ of prescribed dose was also limited to less than $1 \%$ of target volume. When the objectives of PTV were met, the objectives of OARs were determined in the following order of priorities: heart, left lung, contralateral lung.

\section{Planning strategy}

For each patient, the treatment plans were created by using the Eclipse ${ }^{\mathrm{TM}}$ system (Varian Medical System, USA)

Table 1. The optimization objectives used for inverse IMRT/ VMAT planning.

\begin{tabular}{ll}
\hline \multicolumn{1}{c}{ Structure } & \multicolumn{1}{c}{ Objectives } \\
\hline PTV & $\mathrm{V}_{44.6 \mathrm{~Gy}}<1 \%, \mathrm{~V}_{42.5 \mathrm{~Gy}}>95 \%, \mathrm{~V}_{40.4 \mathrm{~Gy}}>100 \%$ \\
Heart & $\mathrm{V}_{10 \mathrm{~Gy}}<20 \%$ and $\mathrm{V}_{20 \mathrm{~Gy}}<10 \%, \mathrm{D}_{\text {mean }}<5 \mathrm{~Gy}$ \\
Left lung & $\mathrm{V}_{10 \mathrm{~Gy}}<40 \%, \mathrm{~V}_{20 \mathrm{~Gy}}<30 \%$ and $\mathrm{V}_{30 \mathrm{~Gy}}<20 \%$ \\
Contralateral lung & $\mathrm{V}_{10 \mathrm{~Gy}}<10 \%$ \\
\hline
\end{tabular}



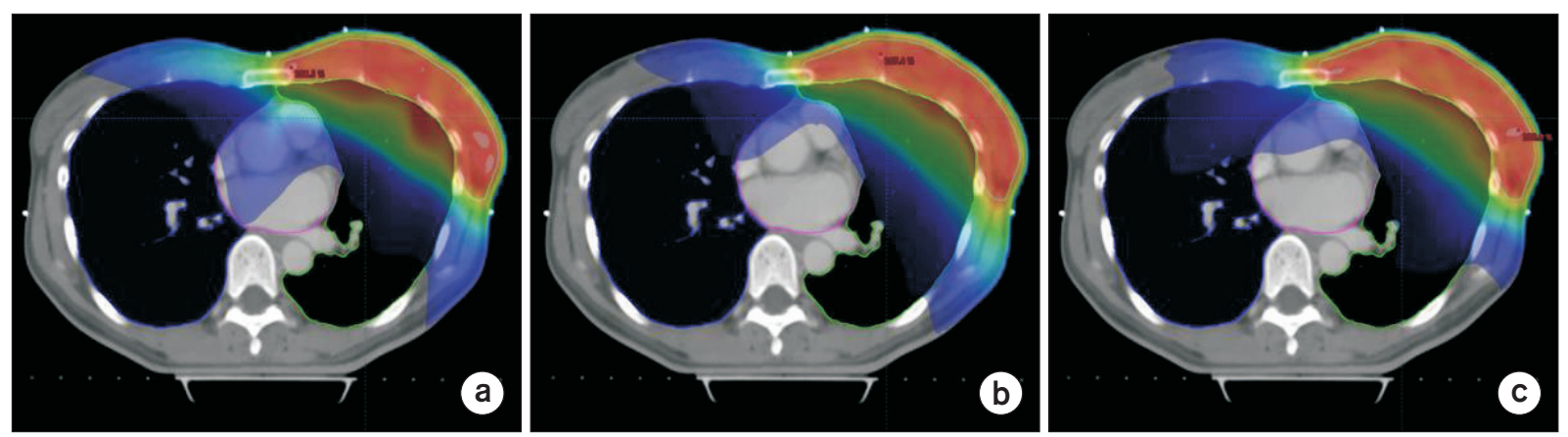

Fig. 1. Example of dose distribution in transverse plane for (a) 1pVMAT, (b) 2pVMAT, and (c) 2tVMAT.

for three VMAT techniques as shown in Fig. 1: one partial arc VMAT (1pVMAT), two partial arcs VMAT (2pVMAT), and two tangential arcs VMAT (2tVMAT). Beam angle arrangements for each plan were slightly different according to PTV position and shape. Each beam was selected proper angles to meet the target coverage and avoid the collision with contralateral breast. First of all, 1pVMAT was generated using a single arc which ranges around $230^{\circ}$ (range, $215^{\circ}$ to $245^{\circ}$ ) to cover the entire treatment area. 2pVMAT was employed with two arcs which has the same arc range used in 1pVMAT. 2tVMAT was generated by using avoidance sector around $60^{\circ}$ (range, $55^{\circ}$ to $65^{\circ}$ ) under the same arc range used in 2pVMAT. The start and stop gantry angles of the avoidance sectors which were identified on a patient-by-patient based on their anatomy were manually set from $0^{\circ}$ to $60^{\circ}$. All plans were used $6 \mathrm{MV}$ photon beam of VitalBEAM ${ }^{\mathrm{TM}}$ with the Millennium $120^{\mathrm{TM}}$ MLC (Varian Medical System, USA). The same set of optimization goal for three type techniques was applied to accomplish fair comparison; hence, the observable discrepancies were mostly ascribed to the disparities of three VMAT techniques. In addition, the photon optimizer (PO, Ver. 13.7, Varian Medical Systems, USA) was used to optimize, and the dose distributions were calculated by the anisotropic analytic algorithm (AAA, Ver. 13.7, Varian Medical Systems, USA) with a calculation grid size of $2.5 \mathrm{~mm}$.

\section{Data analysis}

In order to analyze the target coverage to PTV and doses to OARs, dose volume histograms (DVHs) for each plan was exported. For target coverage, dosimetric parameters such as $\mathrm{D}_{\max }$ (max dose), $\mathrm{D}_{\text {mean }}$ (mean dose), and $\mathrm{V}_{95 \%}$ (percent volume irradiated by $95 \%$ of the prescription dose) of PTV were evaluated. Homogeneity index (HI), conformity index (CI), and conformation number (CN) of PTV were calculated to evaluate the plan quality. HI was calculated by $\operatorname{Eq}(1)$.

$$
\mathrm{HI}=\frac{D_{2 \%}-D_{95 \%}}{D_{50 \%}} \cdots(1)
$$

Where, $\mathrm{D}_{2 \%}, \mathrm{D}_{95 \%}$ and $\mathrm{D}_{50 \%}$ indicate the dose to $2 \%, 95 \%$, and $50 \%$ volume of the PTV, respectively. The lower HI was considered as a plan which has a more homogeneous target dose. The CI (as defined by the International Commission on Radiation Units and Measurements, report 83) is mathematically defined as:

$$
\mathrm{CI}=\frac{V_{R I}}{T V} \cdots
$$

Where, $\mathrm{V}_{\mathrm{RI}}$ is the volume of the target covered by the reference isodose, and TV is the volume of PTV. CI represents the objective measure of how well the distribution of radiation follows the shape of the target volume. The CI refers to the degree of dose conformity, and it is ideal for the CI to remain close to 1 . The $\mathrm{CN}$ which evaluates the conformity to target dose and the healthy tissue irradiation was calculated as: 
where $\mathrm{TV}_{\mathrm{RI}}$ represents the target volume covered with reference isodose.

For OARs, $\mathrm{D}_{\text {max }}, \mathrm{D}_{\text {mean }}, \mathrm{V}_{20 \%}$, and $\mathrm{V}_{10 \%}$ (dose delivered to $20 \%$ and $10 \%$ volume) of contralateral lung, ipsilateral lung, and heart were evaluated. In order to investigate the radiobiological impact on various OARs, the equivalent uniform dose (EUD) based normal tissue complication probability (NTCP) were calculated using MATLAB software based program. ${ }^{20)}$ The paired Wilcoxon' signed-rank test (SPSS, version 12; SPSS Inc, Chicago, IL) was performed for the statistical measure of the difference in dosimetrical parameters between various VMAT techniques. A $P$-value of $<0.05$ was considered to indicate statistical significance.

\section{Results}

\section{Target coverage}

Fig. 1 shows an example of dose distributions generated by (a) 1pVMAT, (b) 2pVMAT, and (c) 2tVMAT. Table 2 indicates the mean and standard deviation of dosimetrical parameters to PTV for all patients. Among three VMAT techniques, there were significant differences in dosimetrical parameters of PTV such as $\mathrm{D}_{\text {max }}, \mathrm{D}_{\text {mean }}, \mathrm{V}_{95 \%}, \mathrm{HI}, \mathrm{CI}$, and CN. The lowest $\mathrm{D}_{\text {max }}(114.93 \pm 1.83)$ was observed in 2pVMAT, while $\mathrm{D}_{\text {mean }}(103.06 \pm 1.65)$ was much higher than other techniques. Furthermore, the $2 \mathrm{pVMAT}\left(\mathrm{V}_{95 \%}=97.60 \pm 1.25\right)$ technique provided significantly increased PTV dose coverage compared with both 1 pVMAT $\left(\mathrm{V}_{95 \%}=91.86 \pm 3.58\right)$ and $2 \mathrm{tV}$ -

Table 2. Dosimetrical parameters to PTV obtained by three VMAT techniques (mean \pm standard deviation).

\begin{tabular}{|c|c|c|c|c|c|}
\hline \multirow{2}{*}{ Metric } & \multirow{2}{*}{ 1pVMAT } & \multirow{2}{*}{ 2pVMAT } & \multirow{2}{*}{ 2tVMAT } & \multicolumn{2}{|c|}{$P$-value } \\
\hline & & & & 1pVMAT vs. 2pVMAT & 2pVMAT vs. 2tVMAT \\
\hline $\mathrm{D}_{\max }(\%)$ & $118.38 \pm 1.72$ & $114.93 \pm 1.83$ & $115.10 \pm 2.25$ & 0.009 & 0.553 \\
\hline $\mathrm{D}_{\text {mean }}(\%)$ & $102.51 \pm 1.86$ & $103.06 \pm 1.65$ & $102.84 \pm 1.70$ & 0.008 & 0.007 \\
\hline $\mathrm{V}_{95 \%}(\%)$ & $91.86 \pm 3.58$ & $97.60 \pm 1.25$ & $96.65 \pm 1.86$ & 0.005 & 0.009 \\
\hline HI & $0.21 \pm 0.04$ & $0.12 \pm 0.02$ & $0.14 \pm 0.03$ & 0.005 & 0.007 \\
\hline $\mathrm{CI}$ & $1.12 \pm 0.13$ & $1.08 \pm 0.11$ & $1.12 \pm 0.11$ & 0.017 & 0.004 \\
\hline $\mathrm{CN}$ & $0.76 \pm 0.05$ & $0.88 \pm 0.06$ & $0.83 \pm 0.05$ & 0.005 & 0.005 \\
\hline
\end{tabular}

Table 3. Dosimetrical and statistical analysis for the organs at risk according to three VMAT techniques (mean \pm standard deviation).

\begin{tabular}{|c|c|c|c|c|c|c|}
\hline \multirow[b]{2}{*}{ Organ } & \multirow[b]{2}{*}{ Metric } & \multirow[b]{2}{*}{ 1pVMAT } & \multirow[b]{2}{*}{ 2pVMAT } & \multirow[b]{2}{*}{ 2tVMAT } & \multicolumn{2}{|c|}{$P$-value } \\
\hline & & & & & $\begin{array}{l}\text { 1pVMAT vs. } \\
\text { 2pVMAT }\end{array}$ & $\begin{array}{c}\text { 2pVMAT vs. } \\
\text { 2tVMAT }\end{array}$ \\
\hline \multirow[t]{5}{*}{ Ipsilateral lung } & $\mathrm{D}_{\max }(\%)$ & $115.58 \pm 3.81$ & $105.88 \pm 1.96$ & $109.57 \pm 4.08$ & 0.005 & 0.007 \\
\hline & $\mathrm{D}_{\text {mean }}(\%)$ & $32.60 \pm 3.21$ & $28.67 \pm 1.71$ & $30.27 \pm 2.84$ & 0.005 & 0.011 \\
\hline & $\mathrm{V}_{20 \mathrm{~Gy}}(\%)$ & $26.11 \pm 3.34$ & $21.43 \pm 1.11$ & $22.76 \pm 2.33$ & 0.005 & 0.022 \\
\hline & $\mathrm{V}_{10 \mathrm{~Gy}}(\%)$ & $43.13 \pm 6.59$ & $37.49 \pm 3.76$ & $39.75 \pm 4.95$ & 0.005 & 0.017 \\
\hline & NTCP & $0.04 \pm 0.03$ & $0.01 \pm 0.01$ & $0.02 \pm 0.01$ & 0.011 & 0.038 \\
\hline \multirow{6}{*}{$\begin{array}{l}\text { Contralateral } \\
\text { lung }\end{array}$} & $\mathrm{D}_{\max }(\%)$ & $53.02 \pm 8.56$ & $62.51 \pm 11.39$ & $47.49 \pm 10.66$ & 0.114 & 0.028 \\
\hline & $\mathrm{D}_{\text {mean }}(\%)$ & $6.81 \pm 1.65$ & $10.29 \pm 6.94$ & $6.94 \pm 1.13$ & 0.005 & 0.007 \\
\hline & $\mathrm{V}_{20 \mathrm{~Gy}}(\%)$ & $0.04 \pm 0.02$ & $0.41 \pm 0.48$ & $0.12 \pm 0.06$ & 0.080 & 0.655 \\
\hline & $\mathrm{V}_{10 \mathrm{~Gy}}(\%)$ & $1.70 \pm 1.29$ & $4.91 \pm 3.23$ & $1.38 \pm 1.23$ & 0.009 & 0.022 \\
\hline & $\mathrm{V}_{5 \mathrm{~Gy}}(\%)$ & $16.30 \pm 8.17$ & $30.48 \pm 11.89$ & $13.61 \pm 5.48$ & 0.005 & 0.013 \\
\hline & NTCP & $<0.001$ & $<0.001$ & $<0.001$ & - & - \\
\hline \multirow[t]{5}{*}{ Heart } & $\mathrm{D}_{\max }(\%)$ & $112.23 \pm 9.35$ & $89.24 \pm 7.43$ & $97.64 \pm 9.03$ & 0.005 & 0.007 \\
\hline & $\mathrm{D}_{\text {mean }}(\%)$ & $17.79 \pm 4.03$ & $14.66 \pm 1.82$ & $15.98 \pm 3.83$ & 0.012 & 0.185 \\
\hline & $\mathrm{V}_{20 \mathrm{~Gy}}(\%)$ & $7.95 \pm 6.05$ & $3.07 \pm 1.12$ & $6.05 \pm 2.93$ & 0.005 & 0.007 \\
\hline & $\mathrm{V}_{10 \mathrm{~Gy}}(\%)$ & $19.77 \pm 7.02$ & $15.21 \pm 4.05$ & $16.60 \pm 8.51$ & 0.012 & 0.445 \\
\hline & NTCP & $<0.001$ & $<0.001$ & $<0.001$ & - & - \\
\hline
\end{tabular}


MAT $\left(\mathrm{V}_{95 \%}=96.65 \pm 1.86\right)$. The HI was $0.21 \pm 0.04,0.12 \pm 0.02$, and $0.14 \pm 0.03$ for $1 \mathrm{pVMAT}, 2 \mathrm{pVMAT}$, and 2tVMAT, respectively. The doses were more conformal in the 2pVMAT compared to the 1pVMAT and 2tVMAT. The CI was lowest in the 2pVMAT ( $\mathrm{CI}=1.08 \pm 0.11$ ), whereas it was similar for 1pVMAT ( $\mathrm{CI}=1.12 \pm 0.13)$ and 2 tVMAT $(\mathrm{CI}=1.12 \pm 0.11)$. The $\mathrm{CN}$ was higher in the $2 \mathrm{pVMAT}(\mathrm{CN}=0.88 \pm 0.06)$ than other two techniques.

\section{Delivered doses to OARs}

Table 3 indicates the dosimetrical parameters for delivered doses of OARs among the 1pVMAT, 2pVMAT, and 2tVMAT. The $\mathrm{D}_{\text {max }}(105.88 \pm 1.96)$ and $\mathrm{D}_{\text {mean }}(28.67 \pm 1.71)$ to ipsilateral lung in 2pVMAT were significantly lower than those in 1pVMAT and 2tVMAT. The $\mathrm{D}_{\max }(62.51 \pm 11.39)$ and $\mathrm{D}_{\text {mean }}(10.29 \pm 6.94)$ of contralateral lung in $2 \mathrm{pVMAT}$ were also significantly lower than those in 1pVMAT and $2 \mathrm{tV}$ MAT. However, $\mathrm{V}_{20 \mathrm{~Gy}}(21.43 \pm 1.11)$ and $\mathrm{V}_{10 \mathrm{~Gy}}(37.49 \pm 3.76)$ of ipsilateral lung were significantly decreased in 2pVMAT, whereas $V_{10 G y}(4.91 \pm 3.23)$ and $V_{5 G y}(30.48 \pm 11.89)$ of contralateral lung was significantly increased in 2pVMAT compared with 2pVMAT and 2tVMAT. 2pVMAT was decreased significantly in $\mathrm{D}_{\max }(89.24 \pm 7.43)$ of heart compared with 1pVMAT (112.23 \pm 9.35$)$ and 2tVMAT (97.64 \pm 9.03$)$. However, there was no statistically significant difference in $D_{\text {mean }}$ of heart with 2tVMAT ( $P$-value $=0.185) . \mathrm{V}_{\text {20Gy }}$ was significantly decreased in 2pVMAT (3.07 \pm 1.12$)$ compared with 1pVMAT (7.95 \pm 6.05$)$ and 2tVMAT (6.05 \pm 2.93$)$. However, no significant difference in $\mathrm{V}_{\text {10Gy }}$ with 2tVMAT $(P$-value $=0.445)$ was observed. Only the average NTCP value of ipsilateral lung was observed to have a relatively apparent difference than those of the other OARs.

\section{Discussion}

In this study, we compared various VMAT techniques such as 1pVMAT, 2pVMAT, and 2tVMAT for left breast radiation therapy during the course of a hypo-fractionated RT comprising 16 fractions. Dosimetrical parameters of various techniques using 3D-CRT, IMRT, and VMAT in left breast cancer have been evaluated in a large of studies. ${ }^{7,12,15,16)}$ These researches report that the IMRT or VMAT for the dose homogeneity and coverage to target volume was significantly increased compared with 3D-CRT. ${ }^{13-16)}$ In addition, VMAT was superior in both the treatment time and the number of MU compared with IMRT. ${ }^{21)}$ Furthermore, VMAT had apparent advantage in reducing the volume of high dose to target volume and disadvantage in increasing the volume of lower dose. ${ }^{14-16)}$ In this study, with respect to the dosimetrical parameters of PTV, 2pVMAT had obvious advantages on not only the HI but also CI and $\mathrm{CN}$ than other two techniques. The 2pVMAT was also improvement of the homogeneous dose distribution as shown in Fig. 1.

High doses to heart and left ascending coronary artery were decreased with the VMAT technique for left breast cancer. Especially, these are reasonable to consider the potential of VMAT techniques on breast cancer, ${ }^{14)}$ because the mean dose and doses of 25 and 30 Gy to heart have been reported to be associated with the complication of heart. ${ }^{5,9)}$ In the previous study, irradiation of the heart to delivered dose during breast radiotherapy resulted in an increase in the subsequent rate of ischemic heart disease linearly with the mean dose to the heart. ${ }^{9)}$ Doses delivered to the heart $\left(\mathrm{D}_{\text {max }}, \mathrm{D}_{\text {mean }}, \mathrm{V}_{20 \mathrm{~Gy}}\right.$, and $\left.\mathrm{V}_{10 \mathrm{~Gy}}\right)$ in 2pVMAT were the lowest in our study. This meant that the 2pVMAT could achieve the reduction of occurrence probability for the heart disease. The $\mathrm{V}_{20 \mathrm{~Gy}}$ which could be used to predict the radiation pneumonitis risk was decreased in ipsilateral lung with 2pVMAT compared to other techniques. However, increasing the delivered dose to contralateral lung was concurrently observed in 2pVMAT. As expected, the low dose volume $\left(\mathrm{V}_{10 \mathrm{~Gy}}\right)$ was significantly increased in 2pVMAT when comparing with 1pVMAT and 2tVMAT. Because the patients with breast cancer are considered long term survivors, the minimization of the delivered dose to contralateral lung is important. In our study, the $\mathrm{V}_{5 \mathrm{~Gy}}$ and $\mathrm{V}_{\text {10Gy }}$ of contralateral lung for 2pVMAT were approximately $30 \%$ and $5 \%$, although corresponding values for 2pVMAT were higher than two VMAT techniques. Because the dose constraints used in our study were excluded in $\mathrm{V}_{5 \mathrm{~Gy}}$ of the contralateral lung, our study showed relatively high $\mathrm{V}_{5 \mathrm{G} y}$ for all techniques compared with threshold presented in RTOG 1005 protocol. ${ }^{22)}$ However, other dosimetrical results were in the same line with the previous studies. ${ }^{13,23)}$ Fur- 
thermore, these are also unclear that this low dose spreads are associated with clinical complication.

Even though the NTCP differences of OARs were only found in ipsilateral lung, the value is the smallest with $2 \mathrm{pV}$ MAT. No remarkable difference of NTCP for other OARs was observed. This may be due to relatively simple target shape that does not include internal mammary node (IMN) and supra clavicle lymph (SCL).

A limitation of our study was to the small number of patient. It was difficult to provide the fully statistical significance for OARs. For example, we could not confirm the statistical significance of parameters such as $\mathrm{D}_{\text {mean }}$ and $\mathrm{V}_{10 \mathrm{~Gy}}$ to Heart (Table 2). Therefore, for future study, we need to investigate more complex shapes and various sizes of target volume for a large number of patients.

\section{Conclusion}

For three VMAT techniques of left breast cancer, the dosimetrical and radiobiological parameters were estimated in this study. This study founded that the plan quality was generally improved with 2pVMAT and, although not for all analyzed parameters, some dosimetrical parameters showed a significant improvement with 2pVMAT than with 1pVMAT and 2tVMAT. In addition, for radiobiological parameters, the 2pVMAT showed the significant improvement in NTCP of ipsilateral lung. Therefore, this study suggests that the use of 2pVMAT as choice for radiotherapy of left breast cancer may be an attractive option.

\section{Acknowledgements}

This work was supported by Grant No. 02-2014-028 from the Seoul National University Bundang Hospital (SNUBH) Research Fund and by the National Research Foundation of Korea (NRF) grant funded by the Korea government (MSIT) (2018R1D1A1B07049159).

\section{Conflicts of Interest}

The authors have nothing to disclose.

\section{Availability of Data and Materials}

All relevant data are within the paper and its Supporting Information files.

\section{Ethics Approval and Consent to Participate}

The study was approved by the institutional review board (IRB approval number; B-1902/520-106).

\section{References}

1. Fisher B, Anderson S, Bryant J, et al. Twenty-year followup of a randomized trial comparing total mastectomy, lumpectomy, and lumpectomy plus irradiation for the treatment of invasive breast cancer. N Engl J Med 2002; 347(16):1233-1241.

2. Veronesi U, Cascinelli N, Mariani L, et al. Twenty-year follow-up of a randomized study comparing breast-conserving surgery with radical mastectomy for early breast cancer. N Engl J Med 2002;347(16):1227-1232.

3. Munshi A, Pai RH, Phurailatpam R, et al. Do all patients of breast carcinoma need 3-dimensional CT-based planning? A dosimetric study comparing different breast sizes. Med Dosim 2009;34(2):140-144.

4. Pignol J, Olivotto I, Rakovitch E, et al. A multicenter randomized trial of breast intensity-modulated radiation therapy to reduce acute radiation dermatitis. J Clin Oncol 2008;26(13):2085-2092.

5. Bird B, Swain S. Cardiac toxicity in breast cancer survivors: review of potential cardiac problems. Clin Cancer Res 2008;14(1):14-24.

6. Lohr F, El-Haddad M, Dobler B, et al. Potential effect of robust and simple IMRT approach for left-sided breast cancer on cardiac mortality. Int J Radiat Oncol Biol Phys 2009; 74(1):73-80.

7. Schubert LK, et al. Dosimetric comparison of left-sided whole breast irradiation with 3DCRT, forward-planned IMRT, inverse-planned IMRT, helical tomotherapy, and topotherapy. Radiother Oncol 2010;100(2):241-246.

8. Nilsson G, Holmberg L, Garmo H, Duvernoy O, Sjögren I, Lagerqvist B, et al. Distribution of coronary artery stenosis after radiation for breast cancer. J Clin Oncol 2012; 
30(4):380-386.

9. Darby SC, Ewertz M, McGale P, Bennet AM, Blom-Goldman U, Brønnum D, et al. Risk of ischemic heart disease in women after radiotherapy for breast cancer. N Engl J Med 2013; 368(11):987-998.

10. Hurkmans CW, Cho BCJ, Damen E, Zijp L, Mijnheer BJ. Reduction of cardiac and lung complication probabilities after breast irradiation using conformal radiotherapy with or without intensity modulation. Radiother Oncol 2002; 62(2):163-171.

11. Hong L, Hunt M, Chui C, Spirou S, Forster K, Lee H, et al. Intensity modulated tangential beam irradiation of the intact breast. Int J Radiat Oncol Biol Phys 1999;44(5):11551164.

12. Xu XL, Wu H, Han SK. Dosimetry study of intensity modulated radiation therapy for left side breast cancer. Chinese Journal of Radiation Oncology 2006;15(3):192-195.

13. Virén, Tuomas, et al. Tangential volumetric modulated arc therapy technique for left-sided breast cancer radiotherapy. Radiol Oncol 2015;10(1):79.

14. Jin, Guang-Hua, et al. A comparative dosimetric study for treating left-sided breast cancer for small breast size using five different radiotherapy techniques: conventional tangential field, filed-in-filed, tangential-IMRT, multi-beam IMRT and VMAT. Radiol Oncol 2013;8(1):89.

15. Qiu, Jian-Jian, et al. Dosimetric comparison of 3D conformal, IMRT, and V-MAT techniques for accelerated partialbreast irradiation (APBI). Med Dosim 2014;39(2):152-158.

16. Badakhshi H, Kaul D, Nadobny J, Wille B, Sehouli J, Budach V. Image-guided volumetric modulated arc therapy for breast cancer: a feasibility study and plan comparison with three-dimensional conformal and intensity-modulated radiotherapy. Br J Radiol 2013;86(1032):20130515.

17. Munshi, Anusheel, et al. Short tangential arcs in VMAT based breast and chest wall radiotherapy lead to conformity of the breast dose with lesser cardiac and lung doses: a prospective study of breast conservation and mastectomy patients. Australas Phys Eng Sci Med. 2017;40(3): 729736.

18. Fogliata, Antonella, et al. Dosimetric trade-offs in breast treatment with VMAT technique. Br J Radiol 2016;90(1070): 20160701.

19. Whelan, Timothy J, et al. Long-term results of hypofractionated radiation therapy for breast cancer. N Engl J Med 2010;362(6):513-520.

20. Gay HA, Niemierko A. A free program for calculating EUDbased NTCP and TCP in external beam radiotherapy. Phys Med 2007;23:115-125.

21. Liu, Haiyun, et al. Evaluation of 3D-CRT, IMRT and VMAT radiotherapy plans for left breast cancer based on clinical dosimetric study. Comput Med Imaging Graph 2016;54: 1-5.

22. Vicini, F., G. M. Freedman, and J. R. White. A phase III trial of accelerated whole breast irradiation with hypofractionation plus concurrent boost versus standard whole breast irradiation plus sequential boost for early-stage breast cancer. RTOG 1005. 2012.

23. Xi, Dan, et al. Advantages of a technique using two 50 degree arcs in simultaneous integrated boost radiotherapy for left-sidebreast cancer. Sci Rep 2017;7(1):14748. 